POLSKA AKADEMIA NAUK - ZAKEAD BADANIA SSAKOW

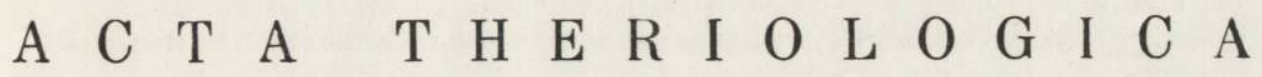
VOL. VII, 1.

\title{
Motorische Kerne des Hirnstammes bei einigen Microtidae (Rodentia)
}

\section{Jądra ruchowe rdzenia przedłużonego niektórych Microtidae (Rodentia)}

\author{
[Mit 30 Photogrammen]
}

I. Einleitung, Material und Methode

II. Bau und Topographie der Kerne

1. Hypoglossuskern (Nucleus nervi hypoglossi) .

2. Die den Hypoglossuskern begleitenden Kerne: .

a. Nucleus sublinqualis sympathicus s. nucleus Rolleri

b. Nucleus praepositus Marburgi.

c. Nucleus intercalatus Staderini .

d. Nucleus paramedianus Jacobsohni

3. Dorsaler parasympathischer Kern der Nerven IX und $X$ (Nucleus dorsalis parasympathicus nervi IX et $\mathrm{X}$ ).

4. Ambiguuskern (Nucleus ambiguus)

5. Facialiskern (Nucleus nervi facialis)

6. Abduzenskern (Nucleus nervi abducentis).

III. Diskussion und Folgerungen

Schrifttum

Tafelbeschreibung

Streszczenie

\section{EINLEITUNG, MATERIAL UND METHODE}

Der Zweck der vorliegenden Arbeit bestand in der Untersuchung des Baues und der Topographie der motorischen Kerne des Hirnstammes bei drei Säugerarten: Erdmaus - Microtus agrestis (L i n n a e u s, 1761), Rötelmaus - Clethrionomys glareolus (S chreber, 1807) und Kleinwuihlmaus, Pitymys subterraneus (D e S ély sLon g ch a m ps, 1835).

Es gibt viele Arbeiten betreffs des Baues des Hirnstammes bei Haustieren: $\mathrm{Ch}$ o$\mathrm{miak}(1951,1953,1954), \mathrm{Ch}$ omiak und Lewandowski (1953), B u jak (1959), Milart (1960), Welento (1956), Vermeulen (1926), Taber (1961) und viele andere. Der Bau dieses Abschnittes des Hirnes bei Haustieren ist folglich ziemlich genau erkannt worden. Eine andere Situation treffen wir in dieser Hinsicht bei Nagern an. Untersuchungen über den Hirnstamm dieser Tiere sind verhältnismässig 
selten durchgeführt worden: Olszewski (1949) und Hoffmann (1957) - sie betreffen das Kaninchen und das Meerschweinchen.

Vorliegende Arbeit betrifft den Bau der motorischen Kerne des Hirnstammes bei anderen Tierarten, die zu derselben Familie gehören. Sie wird das Beweisen des Unterschiedsgrades im Bau dieses Hirnabschnittes bei denjenigen Arten ermöglichen, die zwar der systematischen Ordnung nach nahe aneinander stehen, aber sich jedoch in ihrer Lebensweise unterscheiden. Die erwähnten Arten gehören zu denjenigen Tieren, auf denen man Untersuchungen durchgeführt hat um sie als geeignete Labor-Tiere aufzuzüchten.

Als Untersuchungsmaterial dienten Hirnstämme von den oben erwähnten, geschlechtsreifen drei Arten. Die Tiere wurden mit Chloroform betäubt und danach ausgeblutet. Die herauspräparierten Hirne wurden in Äthylalkohol fixiert und in Paraffin gebettet. Die Blöckchen wurden in Querschnitte in Stärke von $10 \mu$ geschnitten und in Blau von Lauth gefärbt. $\mathrm{Zu}$ Untersuchungen wurden alle Paraffinschnitte verbraucht.

\section{BAU UND TOPOGRAPHIE DER KERNE}

\section{Der Hypoglossuskern (Nucleus nervi hypoglossi)}

Der Kern des Nervs XII der Erdmaus liegt teilweise in dem Ventrikelboden - $1 / 4$ des Kernes, aber teilweise im Hirnstamm ausserhalb des Ventrikels $-3 / 4$. Dieser Kern ist von der unteren Olive kürzer. Sein kaudales Ende liegt etwas nach vorn vom kaudalen Ende der unteren Olive, aber sein vorderes Ende etwas kaudal vom anterioren Ende der Letztgenannten. Im verschlossenen Teil des Hirnstammes liegt der Kern unterhalb des Zentralkanales, dicht an der Naht, ventral vom dorsalen parasympathischen Kern der $I X$ und $X$ Nerven. Nach der Öffnung des Kanales lagert der Kern des Nervs XII ventral von dem Ventrikelboden (Phot. 1). In den $2 / 3$ hinteren des Kernes sind die Zellen lose gelagert und weisen keine deutlichen Anhäufungen auf, aber das vordere 1/3 des Kernes teilt sich auf zwei Anhäufungen auf: die Dorsale (grössere, auf dem Querschnitt abgerundet) und die Ventrale (kleinere, ovale). In der dorsalen Anhäufung treten vorwiegend multipolare, grosse Zellen auf, in der ventralen Anhäufung multipolare Zellen von mittlerer Grösse und dabei spindelförmig. In der Nähe des vorderen Kernendes nähern sich diese Anhäufungen aneinander und vereinigen sich, indem sie eine einheitliche Anhäufung bilden.

Der Kern des Nervs XII ist aus multipolaren grossen Zellen, mittlerer Grösse und aus abgerundeten Zellen aufgebaut. Es treten auch wenige spindelförmige Zellen auf. Der Zellkern ist gross, meistenteils rund und mit intensiv gefärbtem Kernkörperchen (Phot. 7). Die Zellen enthalten eine grosse Anzahl an grobkörnigem Tigroid.

Der Hypoglossuskern der Rötelmaus (Phot. 2) und der Kleinwühlmaus (Phot. 3) ist grundsätzlich ähnlich aufgebaut und gelagert wie bei der 
Erdmaus. Bei der Rötelmaus liegt er gänzlich in dem verschlossenen Teil des Hirnstammes, und bei der Kleinwühlmaus liegen im verschlossenen Teil des Hirnstammes $4 / 5$ des Kernes und im Ventrikelboden 1/5. Ähnlich wie bei der Erdmaus unterliegt der Kern des Nervs XII der übrigen Arten in dem Zentralteil einer Aufteilung. Bei der Rötelmaus treten drei Anhäufungen auf: Die Dorsale (die kleinste mit ovalem Umriss), Die Laterale (die grösste dabei rund) und die Ventrale (oval) - (Phot. 4). Bei der Kleinwühlmaus treten derer zwei auf (Phot. 5) - die Laterale (die kleinere von abgerundeter Gestalt) und die Mediale (die grössere, senkrecht oval). In der Hälfte des Kernes verschiebt sich die laterale Anhäufung nach oben und lagert sich über der medialen Anhäufung. Das hintere und vordere Ende des Kernes des Nervs XII sind bei der Rötelmaus und der Kleinwühlmaus einheitliche Anhäufungen.

Der Kern des Nervs XII ist bei der Rötelmaus und der Kleinwühlmaus aus ähnlichen Zellen aufgebaut wie der Kern desselben Nervs bei der Erdmaus.

\section{Die den Hypoglossuskern begleitenden Kerne}

a. Nucleus sublinqualis sympathicus s. nucleus Rolleri

Der Rollersche Kern ist bei der Erdmaus eine sehr kurze Zellenanhäufung, die im Boden des Ventrikels IV liegt. Nur ein sehr kleiner, kaudaler Abschnitt dieses Kernes liegt im verschlossenen Teil des Hirnstammes. Der Rollersche Kern liegt ventral vom Hypoglossuskern (Phot. 1). Frr besteht aus einer länglichen nicht dicht gelagerten Zellenanhäufung. Auf dem Querschnitt hat er die Gestalt einer ovalen lateral abgeflachten Zellengruppe.

Die den Kern bildenden Zellen sind von mittlerer Grösse, überwiegend von abgerundeter Gestalt (Phot. 8). In ihm treten auch unzahlreiche multipolare Zellen auf. Sie haben einen grossen, runden oder ovalen Zellkern mit einem sich intensiv färbenden Kernkörperchen. Das Tigroid tritt in Gestalt von kleinen, feinen zahlreichen Körnern auf.

Der Rollersche Kern der Rötelmaus (Phot. 2) und der Kleinwühlmaus (Phot. 3) lagert, in Unterscheidung von diesem Kern bei der Erdmaus, gänzlich im verschlossenen Teil des Hirnstammes. Der Aufbau und die Gestalt des Rollerschen Kernes sind grundsätzlich bei allen drei Arten gleich. Dieser Kern ist bei der Rötelmaus und der Kleinwühlmaus aus ähnlichen Zellen wie bei der Erdmaus aufgebaut.

$$
\text { b. Nucleus praepositus Marburgi }
$$

Der Marburgskern der Erdmaus lagert im Boden des Ventrikels IV, nach vorn vom Kern des Nervs XII, in seiner Verlängerung, ventral vom 
dorsalen parasympathischen Kern des $I X$ und $X$ Nerven (Phot. 9). Dic Lage des anterioren Kernendes ist schwer zu bestimmen, denn es dringt in die graue Substanz des Ventrikelbodens ein. Der Marburgskern hat auf dem Querschnitt eine runde aber stellenweise auch ovale Gestalt und ist aus mittleren und kleinen multipolaren Zellen aufgebaut, die genau dicht gelagert sind (Phot. 12). Der Zellkern ist verhältnismässig gross und besitzt ein deutliches Kernkörperchen. Die Zellen enthalten eine zahlreiche Anzahl von grossen Tigroidkörnern.

Auf der Höhe des vorderen Endes der unteren Olive vereinigt sich der Marburgskern mit dem Staderiniskern.

Der Marburgskern der Rötelmaus (Phot. 10) und der Kleinwühlmaus (Phot. 11) sind identisch wie bei der vorhergehenden Art aufgebaut und gelagert.

\section{c. Nucleus intercalatus Staderini}

Der Staderiniskern der Erdmaus ist kurz. Er lagert im Ventrikelboden dorsal von dem Hypoglossuskern und dem Marburgskern und dorsomedial vom dorsalen parasympathischen Kern der $I X$ und $X$ Nerven (Phot. 9). Das kaudale Ende des Kernes befindet sich etwas kaudal vor. dem vorderen Kernende des N. XII, aber das vordere Ende vereinigt sich mit dem Marburgskern. Der Staderinische Kern hat auf dem Querschnitt die Gestalt eines Dreieckes, das mit seiner Basis in der Richtung des Ventrikels IV gewendet ist, aber mit dem Gipfel in lateraler Richtung.

Der Kern ist aus multipolaren, abgerundeten, mittleren und kleinen, dicht gelagerten Zellen aufgebaut (Phot. 13). Der Zellkern ist rund und hat ein deutliches Kernkörperchen. Die Zellen besitzen kleine Mengen von Tigroid in Gestalt von feinen Körnern. Der Staderiniskern der Kleinwühlmaus (Phot. 11) hat einen ähnlichen Aufbau, Gestalt und Lagerung wie der Kern bei der Erdmaus.

Bei der Rötelmaus (Phot. 10) lagert der Staderiniskern teilweise in: Ventrikelboden: ungefähr 1/4 des Kernes, aber teilweise im Hirnstamm ausserhalb des Ventrikels - ungefähr $3 / 4$ des Kernes, dorso-lateral vom Kern des N. XII. Ähnlich wie bei der Erdmaus, ist der Staderinische Kern der Rötelmaus und der Kleinwühlmaus aus multipolaren und abgerundeten Zelien mittlerer Grösse aufgebaut.

\section{d. Nucleus paramedianus Jacobsohni}

Den Jacobsohnskern habe ich bei der Erdmaus nicht festgestellt.

Der Jacobsohnskern der Rötelmaus besteht aus einem kurzen Zellstreifen. Er lagert gänzlich im Hirnstamm ausserhalb des Ventrikels IV, ven- 
tral von der anterioren $1 / 2$ des Kernes des N. XII (Phot. 2). Das kaudale Ende des Kernes erscheint ungefähr in der halben Länge des Hypoglossuskernes, das kraniale Ende reicht vom diesem vorderen Kernende etwas kaudal. Der Jacobsohnskern hat auf dem Querschnitt die Gestalt eines horizontalen Ovales. Der kaudale Abschnitt des Kernes ist von der Naht etwas entfernt, der vordere dagegen weist die Tendenz zur Vereinigung mit dem Kern der gegenüberliegenden Seite auf.

Der Jacobsohnskern besteht aus einem Streifen von multipolaren Zellen von mittlerer und kleiner Grösse und ausserdem aus spindelförmigen Zellen, die sich intensiver färben als der Zellkern des Hypoglossuskernes (Phot. 14). Das Tigroid tritt in Gestalt von zahlreichen feinen Körnern auf.

Bei der Kleinwühlmaus lagert der Jacobsohnskern im Ventrikelboden, ventral vom Kern des N. XII (Phot. 6). Er ist aus Zellen von kleiner aber auch mittlerer Grösse aufgebaut.

\section{Der dorsale parasympathische Kern der Nerven $I X$ und $X$ (Nucleus dorsalis parasympathicus $n n$. IX et X)}

Der dorsale parasympathische Kern der Nerven $I X$ und $X$ lagert bei der Erdmaus teilweise im Ventrikelboden - ungefähr 1/4 des Kernes, teilweise im Hirnstamm ausserhalb des Ventrikels - ungefähr 3/4. Das kaudale Ende dieses Kernes erscheint ungefähr auf der Höhe des kaudalen Poles der unteren Olive. Das vordere Ende reicht bedeutend weiter anterior als das vordere Ende der unteren Olive. Es lagert etwas kaudal von dem vorderen Ende des Staderiniskernes, aber es ist schwer zu bestimmen, denn es dringt in die graue Substanz des Ventrikelbodens ein. Im verschlossenen Teil des Hirnstammes lagert der Kern über dem Zentralkanal, dicht an der Naht. Er weist die Tendenz zur Vereinigung mit dem Kern von der gegenüberliegenden Seite auf. In der Hälfte seiner Kernlänge lagert er kaudal vom Zentralkanal. Im Ventrikelboden liegt er über dem Kern des N. XII (Phot. 1), und weiter nach vorn über dem Marburgischen Kern. Der dorsale parasympathische Kern der Nerven $I X$ und $X$ ist ein langer einheitlicher Zellstreifen. Er besitzt die Gestalt einer Keule, die mit dem dünneren Ende kaudal gerichtet ist. Ihr kaudaler Abschnitt ist auf dem Querschnitt rund, aber der vordere hat den Umriss eines horizontalen Ovales. Der kaudale Kernabschnitt ist aus einer geringen Anzahl von nicht besonders eng gelagerten Zellen aufgebaut. Nach vorn vergrössert sich die Zellenanzahl und sie lagern enger aneinander.

Der Kern ist aus mittelgrossen und kleinen Zellen aufgebaut aber mit einer Überzahl der mittelgrossen (Phot. 15). Es zeigt sich auch eine gc- 
ringe Anzahl von spindelförmigen Zellen. Der Zellkern ist gross, rund mit einem deutlichen Kernkörperchen. Das Tigroid tritt in Gestalt von einer geringen Anzahl von feinen Körnern auf, die gleichmässig in der Zellplasma zerstreut sind.

Bau, Topographie und Zellenstruktur des dorsalen parasympathischen Kernes der Nerven $I X$ und $X$ sind bei der Rötelmaus (Phot. 2) und der Kleinwühlmaus (Phot. 3) ebenso identisch wie bei der Erdmaus.

\section{Der Ambiguuskern (Nucleus ambiguus)}

Der Ambiguuskern der Erdmaus liegt ventral und lateral vom Hypoglossuskern ungefähr in der halben Entfernung zwischen diesem Kern und der Aussenfläche des Hirnstammes (Phot. 16). Das vordere ${ }^{1 / 3}$ des Kernes lagert im Ventrikelboden, das kaudale $2 / 3$ - im verschlossenen Teil des Hirnstammes.

Der Ambiguuskern ist vom Kern des N. XII kürzer. Sein kaudales Ende befindet sich auf der Höhe des Überganges des $1 / 3$ des medialen Kernes des N. XII in seinen vorderen Teil, sein vorderes Ende dagegen lagert etwas kaudal vom vorderen Ende des Hypoglossuskernes. Der kaudale Kernabschnitt ist vom vorderen dünner. Auf dem Querschnitt hat er einen runden Umriss. Auf einigen Querschnitten lässt sich hier der Mangel an für diesen Kern charakteristischen Zellen beobachten, wảs die Aufteilung des Kernes auf 2-3 undeutliche Gruppen zur Folge hat, die eine hinter der anderen lagern. Nach der Öffnung des Zentralkanales vergrössert sich die Anzahl der Zellen. Sie liegen hier dichter gedrängt als im kaudalen Abschnitt. Der vordere Kernabschnitt hat auf dem Querschnitt einen ovalen Umriss.

Der Ambiguuskern ist aus multipolaren, grossen Zellen aufgebaut (Phot. 17), die grosse Zellkerne mit deutlichen Kernkörperchen und üppiges Tigroid in Gestalt von grossen Körnern haben, die sich überwiegend in der Nähe des Kernes konzentrieren.

Der Ambiguuskern der Rötelmaus (Phot. 18) ist ähnlich wie bei der Erdmaus aufgebaut, aber unterschiedlich von dem letzten lagert er gänzlich im verschlossenen Teil des Hirnstammes. Er ist aus abgerundeten, grossen und mittelgrossen Zellen aufgebaut (Phot. 19). Bei der Kleinwühlmaus (Phot. 20) liegt die Hälfte des Kernes im Ventrikelboden und die andere Hälfte im verschlossenen Teil des Hirnstammes. Er teilt sich anfänglich auf zwei Gruppen: Eine Laterale und eine Mediale, aber später beobachten wir in der Hälfte der Kernlänge eine Aufteilung auf vier Streifen: äorso-lateralen, dorso-medialen, ventro-lateralen und ventromedialen (Phot. 21). Der Ambiguuskern der Kleinwühlmaus ist aus multipolaren, mittelgrossen und kleinen Zellen aufgebaut (Phot. 22). 


\section{Der Facialiskern (Nucleus nervi facialis)}

Bei der Erdmaus lagert der Kern des Nervs VII an der ventro-lateralen Wand des offenen Teiles des Hirnstammes. Der kaudale Pol des Kernes befindet sich etwas anterior vom vorderen Ende der unteren Olive, der vordere etwas kaudal vom kaudalen Ende der oberen Olive. Der Kern des Nervs VII hat die Gestalt einer dicken Spindel, die dorso-ventrai abgeflacht ist. Der kaudale Kernabschnitt ist ähnlich wie der vordere eine einheitliche Anhäufung. Der mittlere Abschnitt, der ungefähr der Hälfte des Kernes gleich ist, zerfällt in einige nicht besonders deutliche, längliche Anhäufungen. Anfänglich kann man hier drei Anhäufungen absondern: Die Laterale, die Mediale und die Intermediale. Diese Anhäufungen teilen sich auf nicht besonders deutliche, sekundäre Gruppen. Nur in der lateralen Anhäufung sind sie etwas deutlicher: Die dorsale Gruppe (kleinere) und die ventrale Gruppe (grössere) - (Phot. 23).

In dem vorderen ${ }^{1 / 4}$ des Kernes verringert sich die Zellenanzahl. Auf dem kurzen Abschnitt erhält sich noch die Einteilung auf die drei erwähnten Hauptanhäufungen, aber das vordere Ende ist eine einheitliche Anhäufung. Der Kern ist aus multipolaren, grossen aber auch einer geringen Anzahl von mittelgrossen Zellen aufgebaut (Phot. 24). Die Zellen sind lose gelagert. Sie kennzeichnen sich durch einen grossen, runden Kern mit intensiv gefärbtem Kernkörperchen und eine grosse Anzahl von grobkörnigen Tigroid.

Der Facialiskern der Rötelmaus (Phot. 25) lagert teilweise im Ventrikelboden - zirka $3 / 4$ des Kernes, und teilweise ausserhalb des Ventrikels - zirka ${ }^{1 / 4}$. Er teilt sich in dem medialen Teil auf zwei Anhäufungen: Die Laterale und Mediale, in denen man nicht besonders deutliche sekundäre Gruppen absondern kann.

Der Facialiskern der Kleinwühlmaus (Phot. 26) hat einen ähnlichen Bau und eine ähnliche Lagerung wie bei der Erdmaus. Die Zellen, die den Facialiskern bilden sind bei allen drei Arten identisch.

\section{Der Abduzenskern (Nucleus nervi abducentis)}

Der Kern des N. VI liegt bei der Erdmaus gänzlich im Ventrikelboden, ventro-medial vom absteigenden Arm des N. VII, etwas unterhalb der Krümmung des Facialiskniees (Phot. 27).

Der Kern zieht sich von der durch das vordere Kernende des N. VII durchgeführten Fläche, ungefähr bis zum Knie dieses Nervs. Der Abduzenskern hat die Gestalt einer kurzen dorso-ventral abgeplatteten Spindel. Er ist eine einheitliche Anhäufung von multipolaren, grossen und einer geringen Anzahl von mittelgrossen Zellen (Phot. 30). Die in der Nähe des 
Facialiskniees liegenden Zellen lagern mit ihren langen Achsen parallel zum Knie. Der Zellkern ist gross, rund mit einem deutlichen Kernkörperchen. Das Plasma füllt eine tigroidale Substanz in Gestalt von grossen, gleichmässig zerstreuten Körnern aus.

Der Abduzenskern der Kleinwühlmaus (Phot. 28) hat einen ähnlichen Bau und eine ähnliche Lagerung wie bei der Erdmaus und ist aus identischen Zellen aufgebaut.

Bei der Rötelmaus (Phot. 29) kann man in dem medialen Teil zwei Anhäufungen absondern - die Dorsale und Ventrale. Die den Kern bildenden Zellen sind ähnlich wie bei der Erdmaus und der Kleinwühlmaus.

\section{DISKUSSION UND FOLGERUNGEN}

Die drei Arten, deren Hirnstamm ich untersucht habe, gehören zu der Familie der Wühlmäuse (Microtidae). Sie treten in ähnlichen Milieus auf, aber führen ein verschiedenes Lebensdasein. Obwohl sie in der systematischen Ordnung nahe aneinander stehen, treten hier gewisse, deutliche Unterschiede im Bau des Hirnstammes auf. Die Begründung dieser Unterschiede kann durch keine konkreten Angaben über Bau und Tätigkeit der durch die Gehirnnerven benervten Organe, die ihren Anfang in den beschrieben Kernen nehmen, bekräftigt werden, denn die Anatomie der durch mich untersuchten Arten ist noch nicht genau kennengelernt worden.

Der Hypoglossuskern ist bei der Kleinwühlmaus am besten entwickelt. Auf Grund der Behauptungen von Kappers (1920) dürfte dies für eine grössere Leistungsfähigkeit der Zungenmuskeln bei dieser Art zeugen. Der Kern des N. XII ist bei der Rötelmaus stark nach hinten verschoben - er liegt gänzlich im verschlossenen Teil des Hirnstammes. Bei den übrigen Arten lagert der vordere Teil des Kernes im Ventrikelboden. Vermeulen (1924) hatte festgestellt, dass bei Tieren mit einem schwächer entwickelten Geschmacksinn der Kern des N. XII mehr kaudal liegt als bei anderen. Auf dieser Grundlage könnte man behaupten, dass die Rötelmaus einen schwächer entwickelten Geschmacksinn hat als die übrigen zwei Arten.

Der Rollersche Kern ist bei der Erdmaus am besten entwickelt. Bei der Rötelmaus lagert er gänzlich hinter dem Ventrikel IV, folglich weiter kaudal als bei den übrigen Arten. Bei der Kleinwühlmaus fehlt es im Rollerschen Kern stellenweise an für diesen Kern charakteristischen Zellen, wodurch er auf 2-3 ziemlich undeutliche Gruppen aufgeteilt ist, die eine über der anderen gelagert sind.

Der Staderiniskern liegt bei der Rötelmaus ähnlich wie der Rollersche mehr kaudal als bei den anderen untersuchten Arten. Er lagert dorso-la- 
teral vom Kern des N. XII, aber bei der Erdmaus und der Kleinwühlmaus - dorso-medial. Bei allen untersuchten Arten vereinigt sich der Staderinische Kern mit dem Marburgskern.

Bei der Erdmaus stellte ich die Anwesenheit des Jacobsohnskernes nicht fest. Bei der Rötelmaus liegt dieser Kern im verschlossenen Teil des Hirnstammes, und bei der Kleinwühlmaus - im Ventrikelboden. Der Jacobsohnskern beider dieser Arten weist die Tendenz zur Vereinigung mit dem Kern der gegenüberliegenden Seite auf.

Der Marburgskern liegt bei allen untersuchten Arten in der Verlängerung des Kernes von N. XII, ventral vom dorsalen parasympathischen Kern der $I X$ und $X$ Nerven. Bei der Erdmaus weist er die Verbindung mit dem Hypoglossuskern auf.

Die Bedeutung der den Kern des N. XII begleitenden Kerne ist noch nicht endgültig aufgeklärt worden, obwohl Brodal (1952) ihnen die Funktion der Bewegungskoordination der Zungenmuskeln zuschreibt. Ich begnügte mich also nur mit der Angabe von Unterschieden im Bau und in der Lagerung dieser Kerne bei den durch mich untersuchten Arten.

Der dorsale parasympathische Kern der Nerven $I X$ und $X$ ist bei allen untersuchten Arten in seinem vorderen Teile besser entwickelt. Es fehlt in ihm an irgendeiner Gruppeneinteilung. Der Funktionscharakter der einzelnen Kernabschnitte ist nicht genau bekannt, folglich enthalte ich mich irgendeiner Schlussfolgerung.

Bei allen untersuchten Arten ist der vordere Teil des Ambiguuskernes besser entwickelt. Die beste Entwicklung dieses Kernes beobachten wir bei der Kleinwühlmaus. Es ist möglich, dass dies bei dieser Art mit der besseren Entwicklung der quergestreiften Muskeln in der Speiseröhre in Verbindung steht als bei der Erdmaus und der Rötelmaus.

Der F'acialiskern hat bei allen durch mich untersuchten Arten einen ähnlichen Bau und Lagerung.

Das kaudale und kraniale Ende des Kernes bestehen aus einer einheitlichen Anhäufung, aber der mediale Teil unterliegt einer Aufteilung auf einige Gruppen. Der Kern des N. VII ist bei der Rötelmaus im Verhältnis zu demselben bei den übrigen Arten kaudal verschoben: ${ }^{1 / 4}$ des kaudalen Teiles des Kernes lagert im verschlossenen Teil des Hirnstammes, aber bei den übrigen Arten liegt der Kern gänzlich im Ventrikrelboden. Einen ähnlichen Aufbau dieses Kernes finden wir bei Haustierên ( $\mathrm{C} \mathrm{h}$ o m i a k, 1951; 1954; Chomiak \& Le wandow ski, 1953).

Der Abduzenskern ist am besten bei der Erdmaus, aber am schwächsten bei der Kleinwühlmaus entwickelt. Die schwache Entwicklung des Kernes von N. VI bei der Kleinwühlmaus steht wahrscheinlich mit ihrer unterirdischen Lebensweise in Verbindung. Der Gesichtssinn hat wohl bei ihr eine kleinere Bedeutung als bei den anderen Arten; folglich sind im 
Zusammenhang damit die den Augapfel bewegenden Muskeln und die diese Muskeln benervenden Nerven schwächer entwickelt. Für diese Tatsache spricht die Rückbildung dieses Kernes bei dem Maulwurf (Chomi a k, 1951).

An dieser Stelle möchte ich dem Leiter des Katheders für Anatomie der Tiere d. Landw. Hochschule, Herrn Prof. Dr. Marian $\mathrm{Chomiak}$ meinen herzlichsten Dank sagen für die erteilten Ratschläge und Hilfe bei der Durchführung meiner Arbeit.

Desgleichen danke ich dem Leiter des Institutes für Säugetierforschung der Poln. Ak. d. Wiss. in Bialowieża, Herrn Prof. Dr. August Dehnel für die gütige Bereitstellung zur Ausnutzung der Experimentalzuchttiere.

\section{SCHRIFTTUM}

1. Brodal, A., 1952: Experimental demonstration of cerebellar connexions from the perihypoglossal nuclei (nucleus intercalatus, nucleus praepositus hypoglossi and nucleus of $\mathrm{R} 011 \mathrm{er}$ ) in the cat. J. Anat. 86/2: 110-129, London.

2. Bujak, A., 1959: Jądra ruchowe rdzenia przedlużonego owcy. Ann. Univ. M. Curie-Skłodowska. Sect. DD, 14, 9: 189-205, Lublin.

3. Ch o mi a k, M., 1951: Jądra ruchowe rdzenia przedłużonego krowy, świni i konia. Ann. Univ. M. Curie-Skłodowska. Sect. C 5, 12: 373-440, Lublin.

4. Ch o mi a k, M., 1954: Topografia i budowa jąder ruchowych rdzenia przedłużonego kozy. Ann. Univ. M. Curie-Skłodowska. Sect. DD 8, 12: 181-219, Lublin.

5. Chomiak, M. \& Lewandowski, M., 1953: Kern des Zungenmuskelnerven - nucleus nervi hypoglossi und des Gesichtnerven - nucleus nervi facialis beim Schaf. Ann. Univ. M. Curie-Skłodowska, Sect. C 8, 4: 89-102, Lublin.

6. Hof $\mathrm{fman}$, G., 1957: Atlas vom Hirnstamm des Meerschweinchens. Teil I: Rautenhirn. Verlag S. Hirzel, Leipzig.

7. K a p pers, A., 1920: Vergleichende Anatomie des Nervensystem. Haarlem. (Zit. nach Chomiak, M., 1951).

8. Mil art, Z., 1958: Jądra przedniej części rdzenia przedłużonego i mostu V arol a, konia. Ann. Univ. M. Curie-Skłodowska, Sect. DD 13, 5: 63-87. Lublin.

9. Olszewski, J. \& Mess e n, H., 1949: Cytoarchitektonischer Atlas des Rautenhirns des Kaninchens. S. Karger, Basel - New York.

10. T a ber, E., 1961: The cytoarchitecture of the brain stem of the Cat. I. Brain stem nuclei of Cat. J. Comp. Neurology, 116, 1: 27-52. Philadelphia.

11. Vermeulen, H. A., 1924: De invloed van het huisdievorden op den bouf der hersenen. Neerl. Tijdsch. Geneesk, 68. (Zit. nach Chomi a k M., 1951).

12. W e le n to, J., 1956: Jądra przedniej części rdzenia przedlużonego i mostu V arol a owcy i kozy. Ann. Univ. M. Curie-Skłodowska. Sect. DD, 11, 3: 56-107, Lublin.

Katheder für Anatomie der Tiere,

Landwirtschaftliche Hochschule,

Lublin, ul. Akademicka Nr. 11. 


\section{TAFELBESCHREIBUNG}

Tafel I.

Phot. 1. Querschnitt des Hirnstammes der Erdmaus auf der Höhe d. vorderen $1 / 4$ des Hypoglossuskernes. v IV - Ventrikel IV, nd - dorsaler parasympathischer Kern der Nerven $I X$ und $X$, nhd - dorsale Anhäufung des Kernes von N. XII, nhv - ventrale Anhäufung des Kernes von N. XII, $\mathrm{nR}$ - Rollerskern. Präparat 70. Vergr. $75 \times$.

Phot. 2. Querschnitt des Hirnstammes der Rötelmaus in der halben Länge des Jacobsohnskernes. nd - dorsaler parasympathischer Kern der Nerven IX und $\mathrm{X}$, nh - Kern des N. XII, nJ - Jacobsohnskern, nR - Rollerskern. Präparat 166 . Vergr. $75 \times$.

Phot. 3. Querschnitt des Hirnstammes der Kleinwühlmaus in der halben Länge des Rollerschen Kernes. cc - Zentralkanal, nh - Kern d. N. XII, nd dorsaler parasympathischer Kern der Nerven $I X$ und $X, n R-$ Rollerskern. Präparat 267. Vergr. $75 \times$.

Phot. 4. Querschnitt des medialen Abschnittes des Kernes von N. XII der Rötelmaus. cc - Zentralkanal, nd - dorsaler parasympathischer Kern der Nerven $I X$ und $X$, nhd - dorsale Anhäufung, nhl - laterale Anhäufung, nhv - ventrale Anhäufung des Hypoglossuskernes. Präparat 163. Vergr. $75 \times$.

Phot. 5. Querschnitt des medialen Abschnittes des Hypoglossuskernes der Kleinwühlmaus, cc - Zentralkanal, nd - dorsaler parasympathischer Kern der Nerven $I X$ und $X, n h m$ - mediale Anhäufung, nhl - laterale Anhäufung des Kernes von N. XII. Präparat 260 . Vergr. $75 \times$.

Phot. 6. Querschnitt des Hirnstammes der Kleinwühlmaus in der halben Länge des Jacobsohnskernes. nd - dorsaler parasympathischer Kern der Nerven IX und X, nh - Hypoglossuskern, nJ - Jacobsohnskern. Präparat 268. Vergr. $75 \times$.

\section{Tafel II.}

Phot. 7. Zellen des Hypoglossuskernes der Erdmaus. Präparat 68. Vergr. $300 \times$.

Phot. 8. Zellen des Rollerskernes der Erdmaus. Präparat 70. Vergr. $300 \times$.

Phot. 9. Querschnitt des vorderen Teiles des Staderiniskernes der Erdmaus. v IV - Ventrikel IV, nd - dorsaler parasympathischer Kern der Nerven $I X$ und $X, n S$ - Staderiniskern, $\mathrm{nM}$ - Marburgskern. Präparat 72. Vergr. $75 \times$.

Phot. 10. Querschnitt des vorderen Teiles des Staderiniskernes der Rötelmaus. v IV - Ventrikel IV, nd - dorsaler parasympathischer Kern der Nerven IX und X, nS - Staderinischer Kern, nM - Marburgskern. Präparat 168. Vergr. $75 \times$.

Phot. 11. Querschnitt einer hinteren Hälfte des Staderinischen Kernes der Kleinwühlmaus. v IV - Ventrikel IV, nd - dorsaler parasympathischer Kern der Nerven $I X$ und $\mathrm{X}, \mathrm{nS}$ - Staderinischer Kern, nM - Marburgskern. Präparat 271. Vergr. $75 \times$.

Phot. 12. Zellen des Marburgskernes der Erdmaus. Präparat 72. Vergr. $300 \times$, 
Tafel III.

Phot. 13. Zellen des Staderinischen Kernes der Erdmaus. Präparat 72. Vergr. $300 \ldots$.

Prot. 14. Zellen des Jacobsohnskernes der Rötelmaus. Präparat 166. Vergr. 300X.

Phot. 15. Zellen des dorsalen parasympathischen Kernes der Nerven IX und $X$, der Erdmaus. Präparat 63 . Vergr. $300 \times$.

Phot. 16. Querschnitt des vorderen Abschnittes des Ambiguuskernes der Erdmaus. na - Ambiguuskern. Präparat 69. Vergr. $75 \times$.

Phot. 17. Zellen des Ambiguuskernes der Erdmaus. Präparat 70. Vergr. $300 \times$.

Phot. 18. Querschnitt des vorderen Teiles des Ambiguuskernes der Rötelmaus. na - Ambiguuskern. Präparat 163 . Vergr. $75 \times$.

\section{Tafel IV.}

Phot. 19. Zellen des Ambiguuskernes der Rötelmaus. Präparat 173. Vergr. $300 \times$.

Phot. 20. Querschnitt des kaudalen Abschnittes des Ambiguuskernes der Kleinwühlmaus. cc - Zentralkanal, nh - Kern des N. XII, na - Ambiguuskern. Präparat 264. Vergr. $75 \times$.

Phot. 21. Querschnitt der vorderen Hälfte des Ambiguuskernes der Kleinwühlmaus. amd - dorso-mediale Anhäufung, ald - dorso-laterale Anhäufung, amv - ventro-mediale Anhäufung, alv - ventro-laterale Anhäufung des Ambiguuskernes. Präparat 267. Vergr. $75 \times$.

Phot. 22. Zellen des Ambiguuskernes der Kleinwühlmaus. Präparat 267. Vergr. $75 \times$.

Phot. 23. Querschnitt des medialen Abschnittes des Kernes von N. VII der Erdmaus. fm - mediale Anhäufung, fi - intermediale Anhäufung, fld dorso-laterale Anhäufung, flv - ventro-laterale Anhäufung des Facialiskernes. Präparat 85 . Vergr. $75 \times$.

Phot. 24. Zellen des Facialiskernes der Erdmauș. Präparat 76. Vergr. $300 \times$.

\section{Tafel V.}

Phot. 25. Querschnitt des mittleren Abschnittes des Kernes von N. VII der Rötelmaus. fm - mediale Anhäufung, $\mathrm{fl}$ - laterale Anhäufung des Facialiskernes. Präparat 167 . Vergr. $75 \times$.

Phot. 26. Querschnitt des kaudalen Abschnittes des Facialiskernes der Kleinwühlmaus. nf - Facialiskern. Präparat 277. Vergr. $75 \times$.

Phot. 27. Querschnitt des mittleren Abschnittes des Abduzenskernes der Erdmaus. v IV - Ventrikel IV, gnf - Genu nervi facialis, nas - Kern des N. VI. Präparat 91 . Vergr. $75 \times$.

Phot. 28. Querschnitt des mittleren Abschnittes des Abduzenskernes der Kleinwühlmaus. v IV - Ventrikel IV, gnf - Genu nervi facialis, nas - Kern des N. VI. Präparat 284. Vergr. $75 \times$.

Phot. 29. Querschnitt des mittleren Abschnittes des Abduzenskernes der Rötelmaus. v IV - Ventrikel IV, gnf - Génu nervi facialis, asv - ventrale Anhäafung, asd - dorsale Anhäufung des Abduzenskernes. Präparat 181. Vergr. $75 \times$.

Phot. 30. Zellen des Abduzenskernes der Erdmaus. Präparat 91, Vergr. $300 \times$. 


\section{STRESZCZENIE}

Jądro nerwu podjęzykowego (nucleus nervi hypoglossi) u wszystkich badanych gatunków ulega w części środkowej podziałowi; u nornika burego (fot. 1) występuje sk'pienie grzbietowe i brzuszne, u darniówki (fot. 3,5 ) - boczne i przyśrodkowe. a u nornicy rudej - trzy skupienia: brzuszne, grzbietowe i boczne (fot. 2, 4). Jądro nerwu XII nornicy rudej, w odróżnieniu od pozostałych gatunków leży całkowicie w części zamkniętej rdzenia przedłużonego. U wszystkich badanych przeze mnie gatunków jest ono zbudowane $\mathrm{z}$ komórek wielobiegunowych dużych i średnich (fot. 7).

Jądro Rollera u wszystkich trzech gatunków jest zbudowane w zasadzie podobnie. Leży ono brzusznie od jądra nerwu XII (fot. 1, 2, 3). U nornicy rudej leży bardziej w tyle niż u pozostałych. Najlepszy rozwój jądra R olle r a obserwujemy u nornika burego. U wszystkich gatunków jądro $\mathrm{R}$ oller a zbudowane jest $\mathrm{z}$ komórek zaokrąglonych średniej wielkości (fot. 8).

Jądro M a r burga u wszystkich trzech gatunków jest zbudowane i ułożone podobnie - leży ono w przedłużeniu jądra nerwu podjęzykowego, brzusznie od jądrá grzbietowego parasympatycznego $\mathrm{nn}$. IX i $\mathrm{X}$ (fot. 9, 10, 11). Zbudowane jest $\mathrm{z}$ komórek wielobiegunowych średnich i małych (fot. 12).

Jądro S t a d erin i e go u nornika burego (fot. 9) i darniówki (fot. 11) układa się grzbietowo od jądra M a r burga i brzusznie przyśrodkowo od jądra grzbietowego parasympatycznego $\mathrm{nn}$. IX i X.

U nornicy rudej (fot. 10) leży ono brzusznie i bocznie od jądra grzbietowego parasympatycznego $\mathrm{nn}$. IX i X i nieco bardziej w tyle w stosunku do tego jądra u pozostałych gatunków. Komórki tworzące jądro $\mathrm{Staderiniego} \mathrm{są} \mathrm{zaokrąglone}$ średniej wielkości i małe (fot. 13).

Jądro J a cobsohn a nornicy rudej (fot. 2) leży w rdzeniu przedłużonym poza komorą IV, brzusznie od jądra nerwu XII. U darniówki (fot. 6) jądro J a c o bs o hn a leży całkowicie w dnie komory IV, również brzusznie od jądra n. XII. U nornika burego nie stwierdziłem obecności jądra J a cobsohna. Jądro J a cobsohna nornicy rudej składa się z komórek wielobiegunowych i wrzecionowatych średnich (fot. 14). U darniówki zbudowane jest z komórek wielobiegunowych małych i średnich, oraz z komórek wrzecionowatych.

Jądro grzbietowe parasympatyczne $\mathrm{nn}$. IX i X u wszystkich badanych gatunków jest zbudowane identycznie. Ma ono kształt maczugi zwróconej cieńszym końcem ku tyłowi. Układa się ono grzbietowo od jądra n. XII, bocznie od kanalu ośrodkowego i komory IV (fot. 1, 2, 3). Zbudowane jest $\mathrm{z}$ komórek zaokrąglonych średniej wielkości i małych, ściśle ułożonych (fot. 15).

Jądro niepewne leży brzusznie i bocznie od jądra nerwu XII mniej więcej w połowie odległości między tym ostatnim a brzuszno boczną ścianą rdzenia. U nornika burego (fot. 16) leży ono częściowo w rdzeniu poza komorą IV, częściowo w dnie komory IV.

U darniówki połowa jądra leży w dnie komory IV, połowa zaś poza nią. Tylna część jądra niepewnego darniówki ma budowę jednolitą (fot. 20), natomiast część przednia, w odróżnieniu od pozostałych gatunków dzieli się na cztery skupienia: grzbietowe boczne i przyśrodkowe i brzuszne boczne i przyśrodkowe (fot. 21). U nornika burego zbudowane jest ono z komórek wielobiegunowych dużych (fot. 17), u nornicy rudej $-\mathrm{z}$ komórek zaokrąglonych dużych i średnich (fot. 19), a u darniówki - z komórek wielobiegunowych średnich i małych (fot. 22). 
Jądro nerwu twarzowego leży w brzuszno-bocznej części rdzenia przedłużonego (fot. $23,25,26$ ). U nornicy rudej tylny odcinek jądra leży w części zamknietej rdzenia przedłużonego. U pozostalych gatunków - całkowicie $\mathrm{w}$ dnie komory IV. W części środkowej jądro rozpada się na kilka grup. U wszystkich gatunków jądro n. VII zbudowane jest z komórek wielobiegunowych dużych (fot. 24).

Jądro nerwu odwodzącego leży u wszystkich badanych gatunków w dnie komory IV, brzusznie od kolanka nerwu VII. U nornika burego (fot. 27) i darniówki (fot. 28) ma ono budowę jednolitą. U nornicy rudej natomiast dzieli się w części środkowej na dwa skupienia: grzbietowe i brzuszne (fot. 29). U wszystkich gatunków jądro n. VI zbudowane jest $\mathrm{z}$ komórek wielobiegunowych dużych i średnich (fot. 30, $42,44)$. sat. III k1. 80 g. Format E5. Cena $10 \mathrm{zl}$.

Białostockie Zakłady Graficzne. Zam. $1314 *$ A-3. 
ACTA THERIOLCGICA Vol. VII, 1.

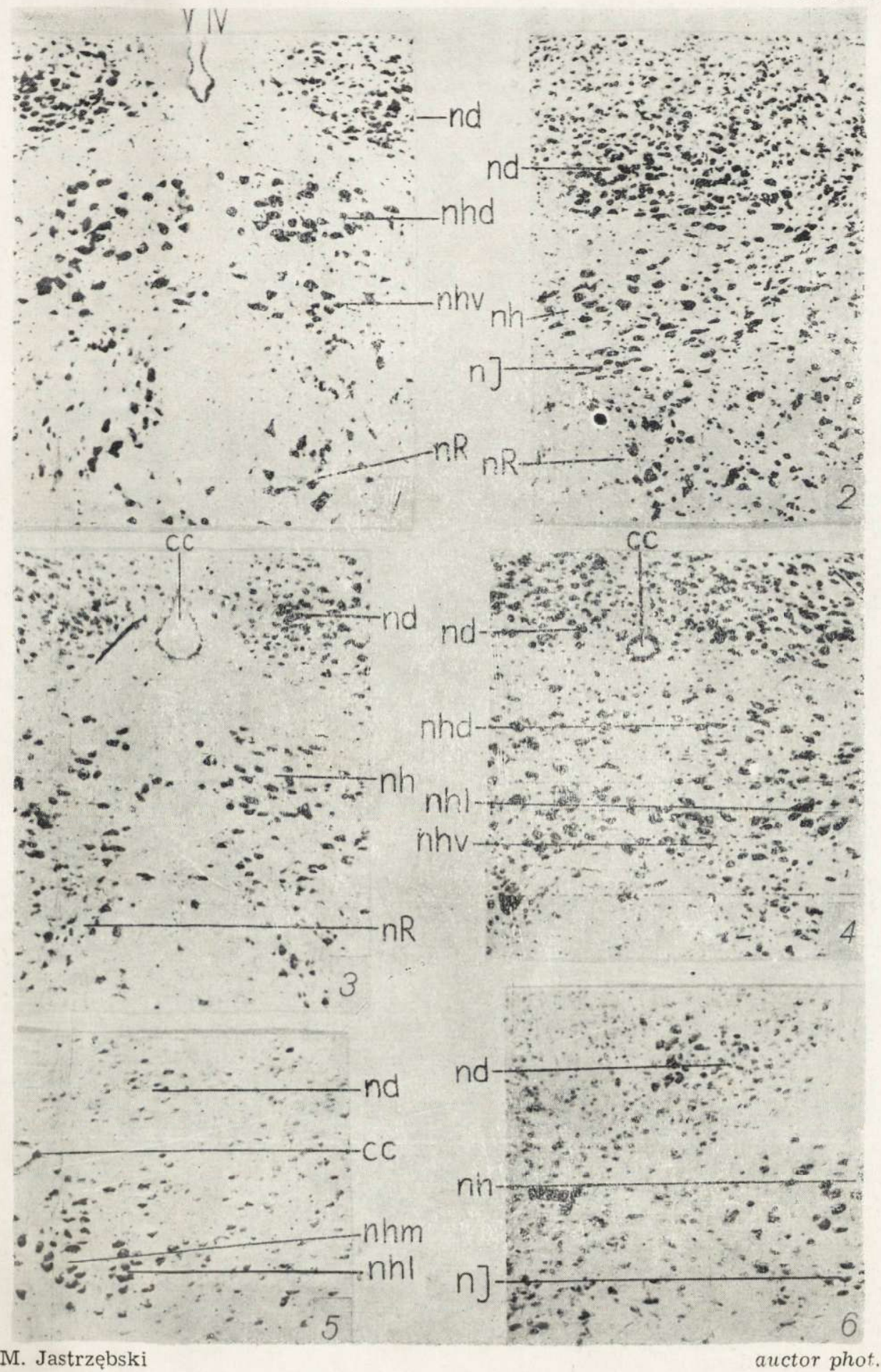


ACTA THERIOLOGICA Vol. VII, 1.

Tafel II.
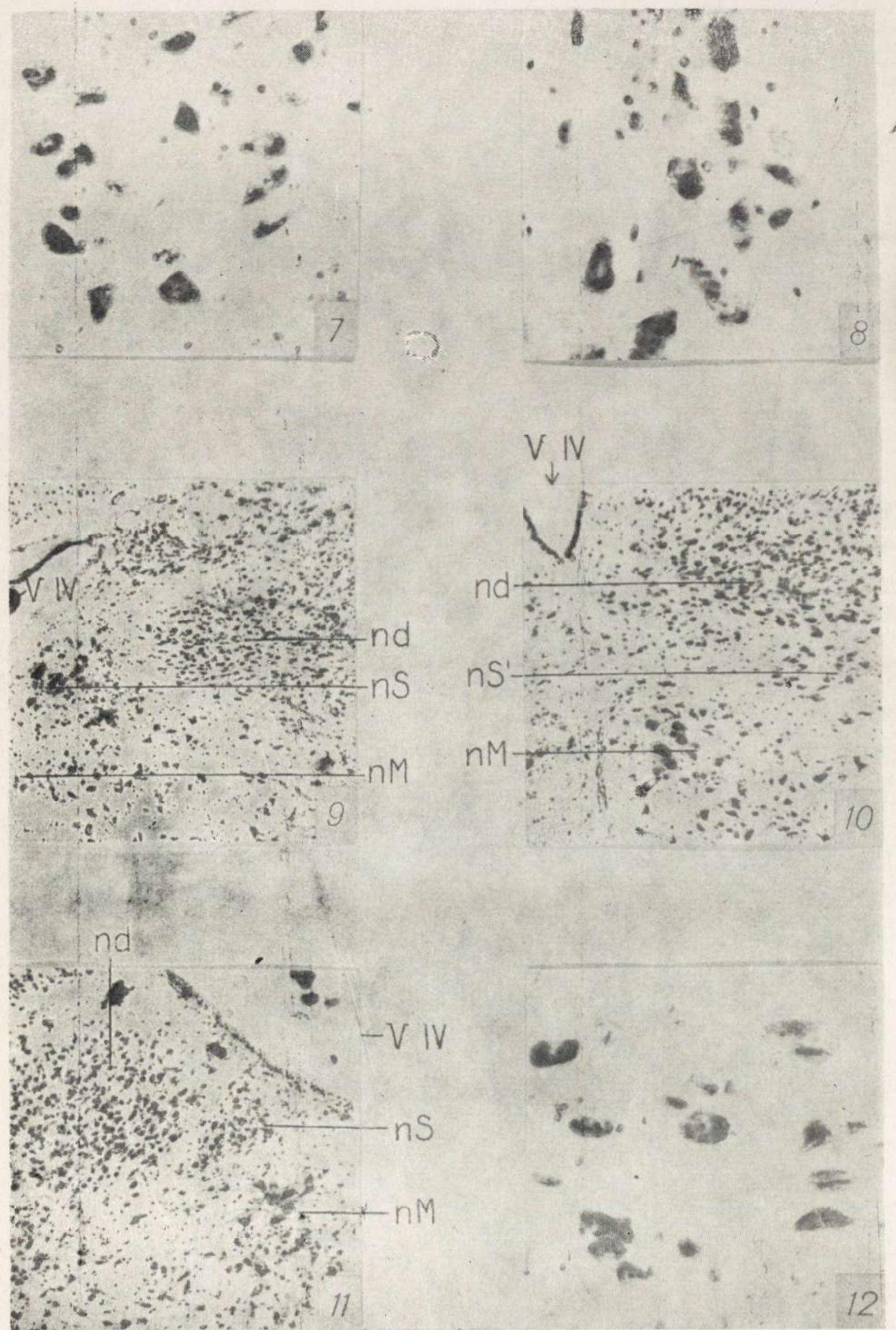

M. Jastrzębski

auctor phot 
ACTA THERIOLOGICA Vol. VII, 1.

Tafel ill,
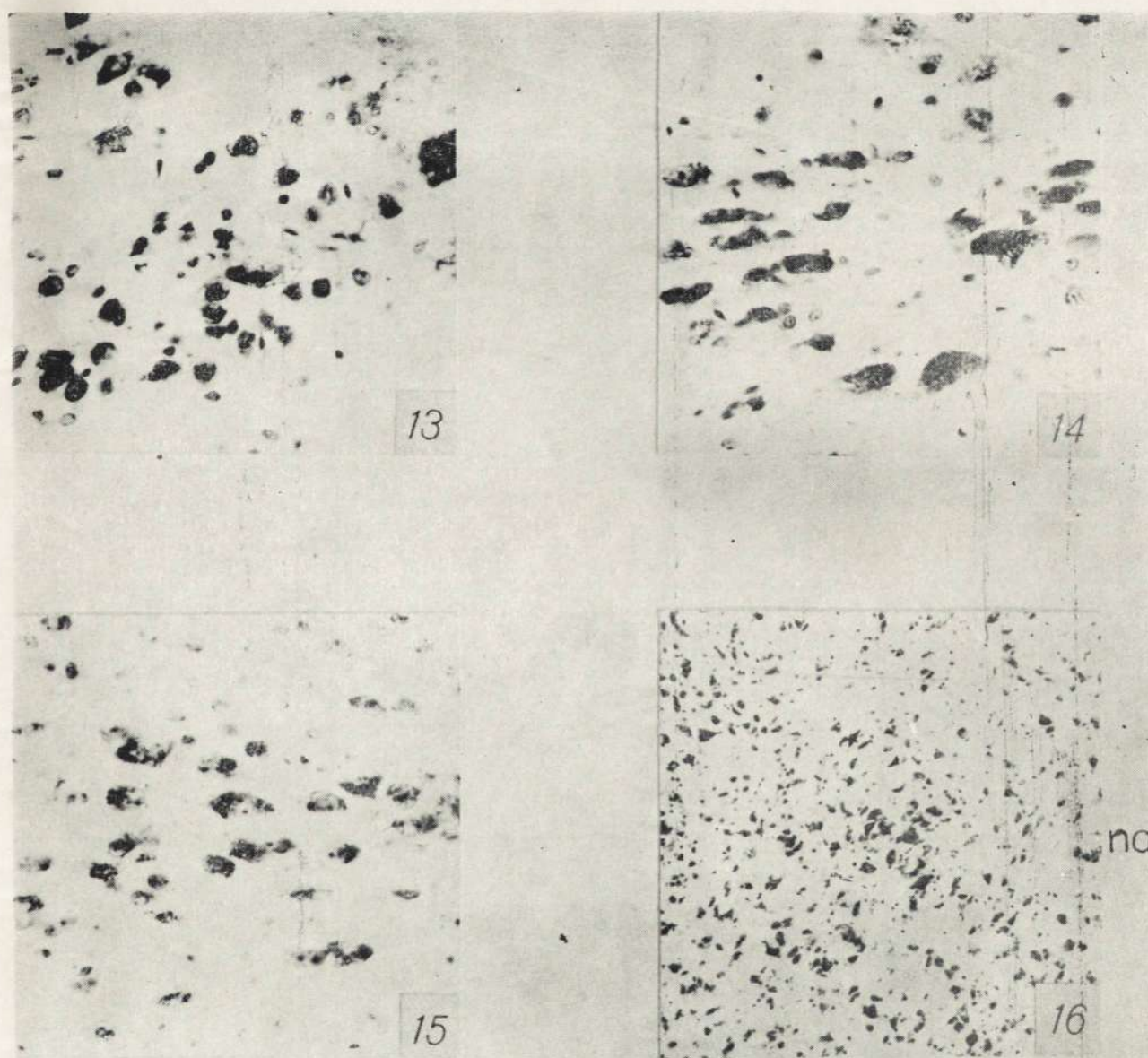

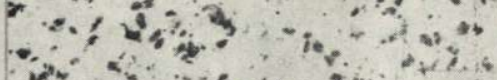

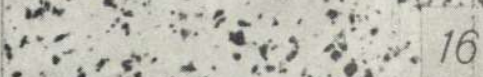
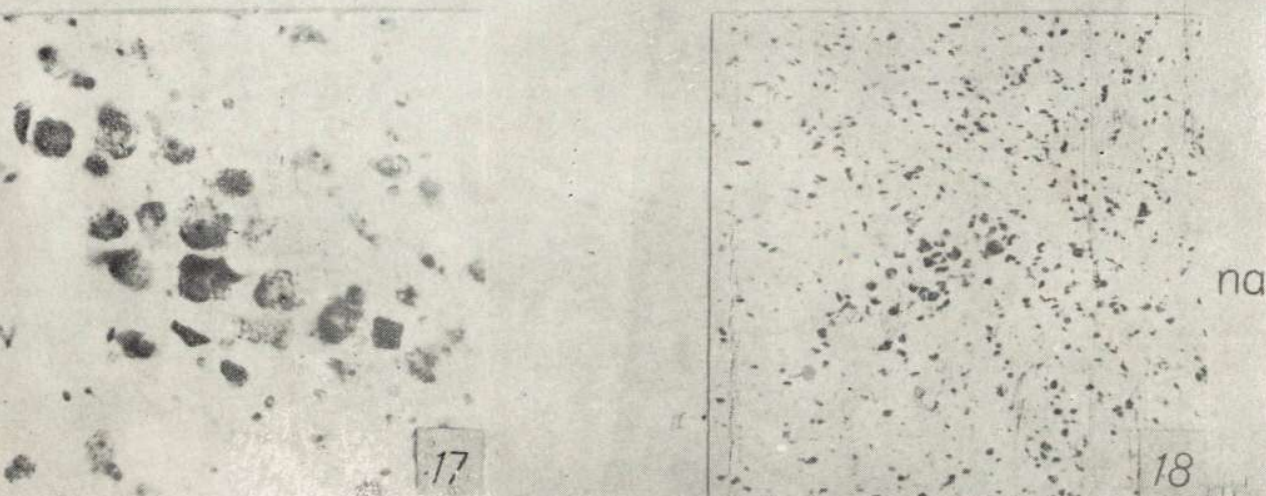

M. Jastrzębski

auctor phot. 
ACTA THERIOLOGICA Vol. VII, 1.
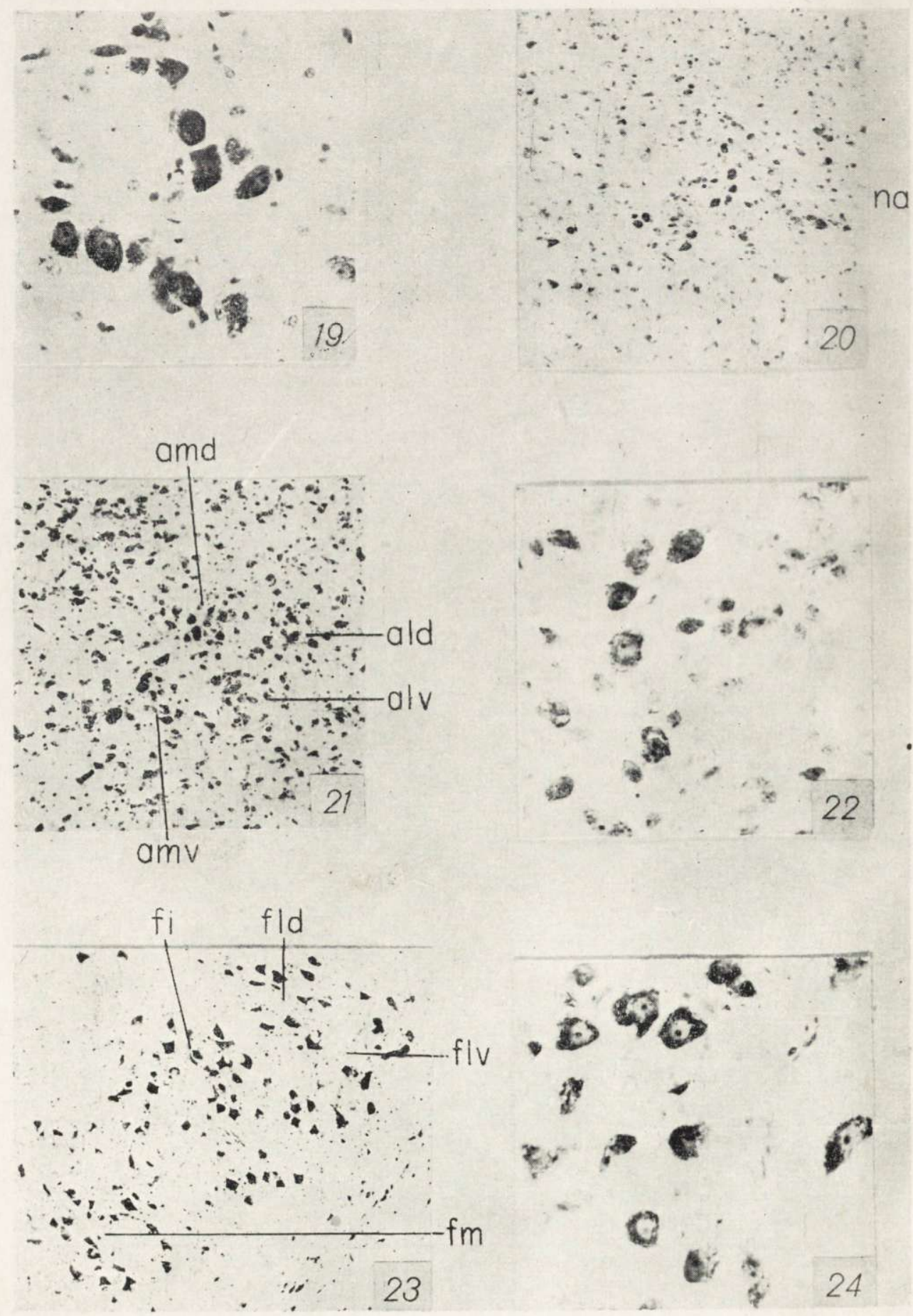

M. Jastrzębski 
AC'T'A THERIOLOGICA Vol. VII, 1.

Tafel $v$.

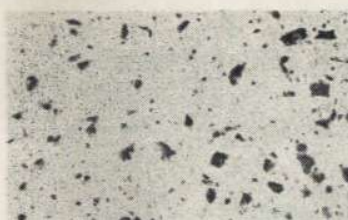

$\sin -\frac{1}{4}$

$\Rightarrow+\infty a^{2}$

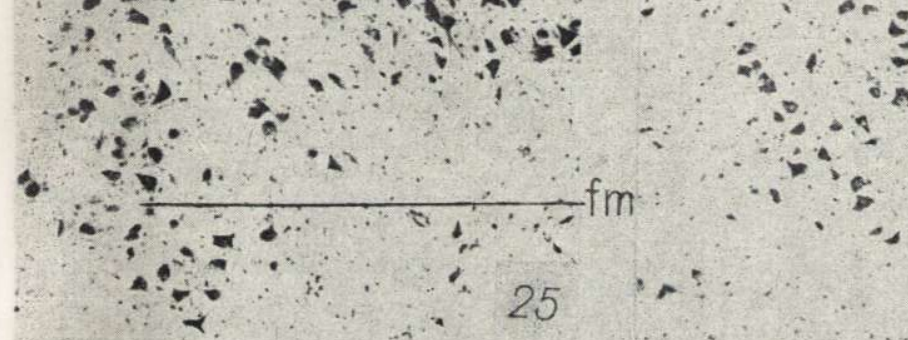

V,IV VIV

$6 x^{2}+4 x^{2}$

$6 x^{2}+4,2$

$x_{1}: y^{2}$

$+e^{\infty}$

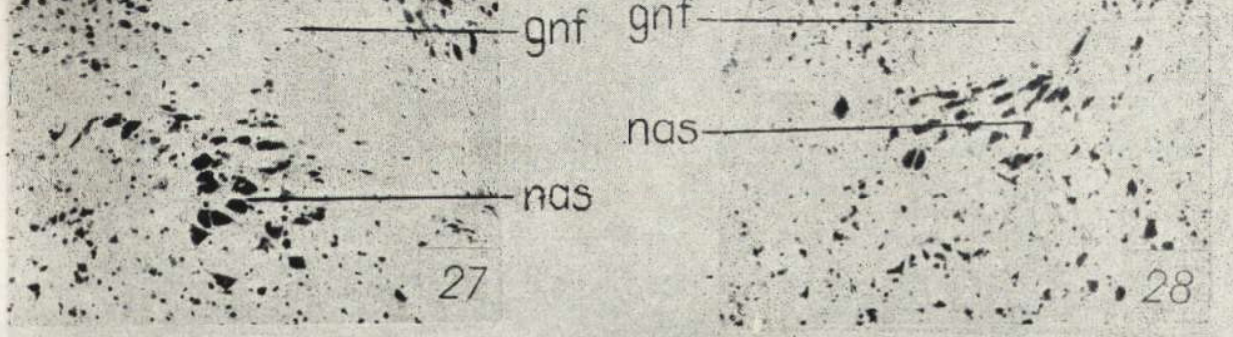

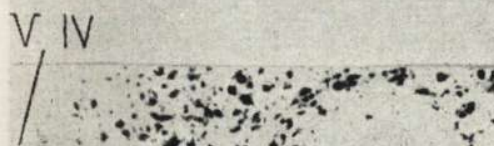

40 -gnf
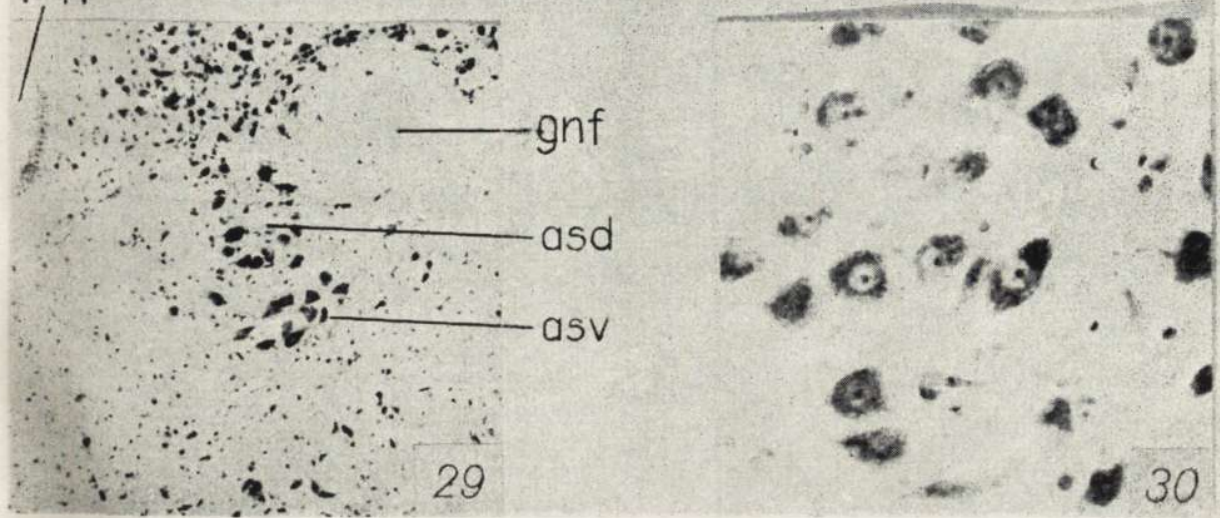

IM. Jastrzębski

auctor phot. 
BIBLIOTEKA

Instytutu Biologii Ssaków

Polskiej Akademii Nauk

$\mathrm{NrCz} .40 .2$ 